\title{
DOAÇÃO DE ÓRGÃOS: ENTENDIMENTO DE ESTUDANTES DOS CURSOS DE MEDICINA E DE ENFERMAGEM
}

\author{
Organ transplantation: understanding of students from the Medicine and Nursing courses
}

\author{
Daniel Sarmento Bezerra1, André Ricardo Bezerra Bonzi', Ivanice Bezerra da Silva Gomes', José Gustavo Sampaio de Sá1, \\ Tânia Regina Ferreira Cavalcanti ${ }^{3}$, Waléria Bastos de Andrade Gomes Nogueira ${ }^{3}$
}

\section{RESUMO}

Objetivo: A pesquisa teve como objetivo discutir o entendimento de alunos dos cursos de Enfermagem e Medicina sobre o transplante de órgãos. Método: O presente estudo caracteriza-se como uma pesquisa de campo, descritiva, com abordagem quantitativa, que avaliou o entendimento dos alunos de Enfermagem e Medicina acerca dos transplantes de órgãos. A pesquisa foi realizada nas faculdades Nova Esperança, FAMENE/FACENE e Maurício de Nassau - João Pessoa. A população foi constituída por discentes do $2^{\circ}$ e $8^{\circ}$ períodos de cada curso das instituições de ensino supracitadas. A amostra constituiu-se de cento e vinte (120) desses discentes. $O$ instrumento escolhido para a coleta dos dados foi um questionário estruturado em duas partes, que evidenciava dados de identificação dos participantes e dados relacionados ao conhecimento de transplantes de órgãos. A coleta de dados, após aprovação do projeto pelo Comitê de Ética e Pesquisa FACENE/FAMENE, foi realizada durante o mês de outubro de 2015. Os dados foram analisados e tabulados estatisticamente, com o auxílio do software estatístico SPSS (Versão 18). A pesquisa respeitou os aspectos éticos preconizados pela Resolução CNS 466/2012, como também, pela Resolução 1931/2009 CFM, Capítulo XII. Resultados: A pesquisa verificou que aproximadamente $50 \%$ dos alunos não possuem conhecimento suficiente a respeito do diagnóstico de morte encefálica e que $85,5 \%$ não têm conhecimento sobre o Sistema Nacional de Transplantes, implicando, assim, numa dinâmica negativa em relação ao processo do transplante de órgãos. O resultado do estudo sugere que as faculdades reavaliem o conteúdo ministrado. Conclusão: A desinformação sobre o assunto faz com que milhares de profissionais não se interessem pela área e não se envolvam na causa, contribuindo, assim, para a perda de órgãos viáveis e, consequentemente, para a morte de pessoas que poderiam ser tratadas.

Descritores: Transplante de Órgãos; Estudantes de Medicina; Estudantes de Enfermagem; Coleta de Tecidos e Órgãos.

Instituições:

${ }^{1}$ Curso de Medicina da Faculdade de Medicina Nova EsperançaFAMENE - João Pessoa/PB - Brasil.

${ }^{2}$ Curso de Enfermagem da Faculdade Maurício de Nassau - João Pessoa/PB - Brasil.

3 Departamento de Medicina da Faculdade de Enfermagem Nova Esperança- FACENE, João Pessoa/PB - Brasil.

\section{Correspondência:}

Daniel Sarmento Bezerra

Avenida Sergipe, 311 - CEP 58030-190, João Pessoa/PB - Brasil

Tel.:(83) 99694-3834 / 99921-4331

E-mail: sarmentomeddaniel@gmail.com

Recebido em: 17/01/2016

Aceito em: 19/02/2016

\section{INTRODUÇÃO}

Os primeiros transplantes realizados no Brasil datam da década de 60. O Sistema Nacional de Transplantes (SNT) foi criado pelo Decreto $N^{\circ} 2.268$ de 30 de junho de 1997, como entidade responsável pelo controle e monitoramento do processo de doação de órgãos e tecidos e transplantes realizados no país, com o objetivo de desenvolver o processo de captação e distribuição de tecidos, órgãos e partes retiradas do corpo humano para fins terapêuticos. ${ }^{1-3}$

A doação de órgãos pode ser realizada entre vivos (parentesco até quarto grau), através de doador falecido e mantido estável em UTI (Unidade de Terapia Intensiva) e também, doador falecido, no caso da 
Daniel Sarmento Bezerra, André Ricardo Bezerra Bonzi, Ivanice Bezerra da Silva Gomes, José Gustavo Sampaio de Sá,

Tânia Regina Ferreira Cavalcanti, Waléria Bastos de Andrade Gomes Nogueira

doação de córneas. ${ }^{2}$ Outrossim, existe constante e crescente demanda por transplantes de órgãos em todo território nacional e sabemos que, tanto a carreira do médico quanto a do enfermeiro, não contempla em seus currículos nenhuma matéria voltada especificamente para esse tema, o que contribui para que ele seja negligenciado pelos profissionais de saúde supracitados, havendo a necessidade de buscar, por conta própria e interesse, cursos e especializações sobre transplantes de órgãos, na forma extracurricular. ${ }^{4}$

Dentro do ambiente hospitalar, em urgências e unidades de terapia intensiva, há desconhecimento por parte de muitos médicos e enfermeiros, dos diversos procedimentos que envolvem o transplante de órgãos, tais como: abertura de protocolo de morte encefálica, manutenção do paciente com morte encefálica, notificação desses pacientes e a existência de convênios com a Central de Transplantes regional. ${ }^{4}$

A doação de órgãos deve dar-se de forma gratuita, inclusive, não cabendo indenização em caso de cancelamento. O paciente deve estar devidamente registrado no serviço hospitalar e não apresentar hipotermia (temperatura abaixo de $35^{\circ}$ ), a causa do óbito deve ser conhecida e não causada por neoplasias. Ademais, o responsável pelo corpo é o parente - de até $2^{\circ}$ grau - que deverá decidir se doa ou não os órgãos do parente falecido (córneas); ou em morte encefálica onde ocorre ausência de perfusão sanguínea cerebral ou ausência de atividade elétrica cerebral ou ausência de atividade metabólica cerebral (doação multi-órgãos - órgãos sólidos), sendo os seguintes tecidos humanos excluídos da lista: óvulos, sangue e esperma. Além disso, o diagnóstico de morte encefálica, constatada e registrada por dois médicos não participantes das equipes de remoção e transplante e regulamentada pelo Conselho Federal de Medicina. ${ }^{1,5,6}$

O transplante obedece a uma listagem nacional, onde o paciente é cadastrado pelo respectivo médico que o acompanha clinicamente e aguarda por uma doação compatível com sua etiologia ou condição clínica. A captação dos órgãos é feita em estabelecimentos cadastrados e por equipe treinada, que possua certificação para tais atividades. O registro dessas equipes e do estabelecimento onde os procedimentos médico-cirúrgicos ocorrem possuem prazos rigorosamente executados. ${ }^{2}$

Por outro lado, temos a atuação dos profissionais de saúde: o enfermeiro deve estar apto a reconhecer os sinais de uma provável morte encefálica, informar ao médico plantonista e tomar as ações corretas para a manutenção hemodinâmica do paciente. Além disso, deve informar a CIHDOTT (Comissão Intra-Hospitalar de Doações de Órgãos e Tecidos para Transplantes).
Logo, esse profissional possui função determinante na conduta da doação. ${ }^{7}$

Ao mesmo tempo, o médico, deve atentar para os sinais e sintomas dos pacientes internados em ambientes de urgência e emergência, no intuito de corroborar com a identificação e correto manejo dos prováveis doadores de órgãos, inclusive, mantendo postura ética perante todos os procedimentos e protocolos que viabilizam o transplante de órgãos. ${ }^{7}$

Profissionais de saúde ainda não possuem ideia formada sobre o conceito de morte encefálica - decisivo na doação de órgãos - existindo muitas contradições e confusões acerca do diagnóstico desta. Médicos intensivistas que trabalham em UTI - têm demonstrado que possuem pouco conhecimento acerca dos protocolos e métodos que devem ser empregados ao identificar um provável doador de órgãos e, por consequência, muitas doações são negligenciadas e mal geridas. ${ }^{7}$ A má informação da população e a educação insuficiente dos profissionais de saúde na graduação concorrem para que haja impasse no desenvolvimento dos transplantes. ${ }^{8}$

Nesse sentido, surgiu o interesse em pesquisar e discutir o entendimento apresentado por alunos dos cursos de Enfermagem e Medicina sobre os transplantes de órgãos.

\section{MATERIAL E MÉTODO}

O presente estudo caracterizou-se como uma pesquisa de campo, descritiva, com abordagem quantitativa, que avaliou o entendimento dos discentes dos cursos de Medicina e de Enfermagem, sobre doação e transplante de órgãos. É válido ressaltar que não houve qualquer tipo de atividade, palestra, aula, ou outro método educacional acerca da temática pesquisada neste trabalho antes ou depois da pesquisa. O estudo teve como objetivo identificar o entendimento dos entrevistados diante da vivência de cada um deles até o momento em que foi aplicado o questionário. A coleta de dados envolveu duas Instituições de Ensino Superior - IES, no município de João Pessoa, que oferecem respectivamente, os cursos de Medicina/Enfermagem e Enfermagem: Faculdades de Medicina e de Enfermagem Nova Esperança e Faculdade Maurício de Nassau João Pessoa.

A escolha dessas IES deu-se em virtude dos pesquisadores serem discentes dos referidos cursos em cada uma delas. Foram entrevistados discentes do $2^{\circ}$ e $8^{\circ}$ períodos de ambos os cursos, em ambas as faculdades, respeitando a oferta de cursos de cada uma. A amostra constituída totalizou cento e vinte (120) desses discentes; sendo trinta (30) de cada período. Teve como critério de inclusão: estudantes 
maiores de 18 anos, propriamente matriculados e que concordassem em participar, mediante assinatura ndo Termo de Consentimento Livre e Esclarecido - TCLE.

O instrumento escolhido para a coleta de dados foi um Questionário estruturado em duas partes: Parte I - Dados de identificação dos participantes; e Parte II - Dados relacionados à temática, conhecimento de transplante de órgãos. A escolha do questionário visou poupar tempo, obter dados de fácil compreensão e ser discreto, não inibindo a opinião sincera do entrevistado.

\section{RESULTADO}

A presente pesquisa teve início a partir da explicação dos próprios objetivos e da abordagem dos alunos dos cursos de Enfermagem e Medicina. Os alunos das instituições pesquisadas foram escolhidos ao acaso, sendo que, no total, 120 indivíduos foram entrevistados e apenas 17 recusaram-se a responder ou estavam ausentes na hora da pesquisa, totalizando 103 discentes.

A distribuição dos questionários obteve a seguinte disposição: a turma do segundo período de Medicina foi representada por 29 estudantes $(96,67 \%)$, a do segundo período de Enfermagem por 30 graduandos (100\%), a do oitavo período de Medicina por 23 alunos (76,66\%) e a do oitavo período de Enfermagem, por 21 estudantes $(70 \%)$.

\section{DISCUSSÃO}

\section{"Você estudou em sua formação acadêmica assuntos como morte encefálica ou transplantes de órgãos?"}

Dentre os entrevistados, $53,39 \%$, relataram ter assistido a cursos, aulas ou palestras sobre temáticas envolvendo o transplante de órgãos e tecidos e $11,5 \%$ responderam positivamente quando abordados sobre o funcionamento e a legislação dos transplantes de órgãos. Ao investigar as regras da diretriz nacional de educação superior em saúde, ficou constatado que não existem citações sobre o transplante de órgãos, o que culmina na não inclusão desses assuntos na grade curricular dos cursos de saúde de Enfermagem e Medicina. ${ }^{9}$

No segundo período do curso de Medicina, apenas cinco dos alunos relataram ter recebido informações, aulas, palestras ou cursos sobre transplantes de órgãos, enquanto, no oitavo período de Medicina, 23 alunos informaram ter tido algum estudo sobre transplantes. Em paralelo, apenas 12 alunos do segundo período de Enfermagem e 12 do oitavo período responderam ter adquirido algum tipo de informação ou estudo relacionado aos transplantes de órgãos.

Esses dados são preocupantes, uma vez que a desinformação contribui para que os futuros profissionais desconheçam o correto manejo do paciente (provável doador) que envolve: instabilidade cardiovascular, perda do equilíbrio metabólico, hipoperfusão tecidual e, a partir daí, preservar os órgãos até que sejam captados, ${ }^{7}$ bem como não incentiva a formação de novas equipes técnicas.

\section{"Você doaria seus órgãos?"}

Em ambos os cursos, $88 \%$ dos alunos declararam-se doadores de órgãos. É válido ressaltar que as razões envolvidas na decisão do entrevistado em doar ou não seus órgãos não foram abordadas neste estudo.

Contudo, um estudo transversal feito no ano de $2010 \mathrm{com}$ 364 estudantes de Medicina, na Universidade Federal de Juiz de Fora, concluiu que as mulheres demonstram maior intenção de doar seus órgãos. ${ }^{10}$ Por outro lado, temos dados que são contraditórios à ideia de que a maioria da população tem a intenção de doar. Em estudo desenvolvido no ano de 2009, em universidade privada da Zona Sul do município de São Paulo, 55\% dos alunos do curso de Enfermagem declararam-se não doadores (o que soa incongruente com a função que exercem), sendo esses dados bem próximos aos encontrados junto à população que não tem relação alguma com a área de saúde. ${ }^{11}$

\section{"Você conhece a legislação brasileira sobre transplante de órgãos?"}

Já quando abordados sobre o SNT, ficou demonstrado que os alunos desta pesquisa não possuem conhecimento satisfatório: 15 alunos (14,5\%) responderam possuir algum conhecimento e 88 alunos $(85,5 \%)$ admitiram não saber nada ou muito pouco a respeito.

\section{CONCLUSÃO}

A literatura médica ainda é pobre e pouco informativa e os estudantes, público mais importante a ser capacitado no sentido de garantir que o transplante ganhe força e seja aceito com menos suspeitas, estão pouco atualizados. O número de transplantes feitos hoje no país é satisfatório, mas existe um potencial muito grande a ser explorado para a captação mais eficiente, além da capacitação de médicos e enfermeiros.

$O$ resultado do estudo sugere que as faculdades reavaliem o conteúdo ministrado, já que os resultados apontam lacunas na educação dos futuros profissionais 
Daniel Sarmento Bezerra, André Ricardo Bezerra Bonzi, Ivanice Bezerra da Silva Gomes, José Gustavo Sampaio de Sá, Tânia Regina Ferreira Cavalcanti, Waléria Bastos de Andrade Gomes Nogueira

de saúde que podem ser preenchidas de forma bastante coerente.

É importante informar que os autores, estão desenvolvendo junto às respectivas secretarias dos cursos que frequentam, projetos que visam atuar de forma permanente e duradoura, no intuito de criar uma cultura para o transplante de órgãos.
A inserção de temáticas, estudos e discussões acerca do transplante de órgãos na grade curricular dos cursos de Enfermagem e Medicina, principalmente, é condição importante para a formação de novos profissionais que atuarão nessa área médica, contribuindo, assim, para um maior número de captações e, consequentemente, o tratamento de milhares de pessoas.

\section{ABSTRACT}

Purpose: The aim of the research was to discuss the knowledge presented by the Nursing and Medical course students on the organ transplantation. Method: This study is characterized as a descriptive field research with quantitative approach assessing the understanding of Nursing and Medical students about organ transplantation. The survey was conducted at the Nova Esperança (FAMENE / FACENE) and Maurício de Nassau Colleges in the city of João Pessoa. The population was composed by students of the 2 nd and 8 th periods of each course of the above mentioned educational institutions. The sample consisted of one hundred and twenty (120) students. The chosen instrument for the data collection was a two-part structured questionnaire that showed the identification data of the participants and data related to their knowledge on organ transplantation. After the project was approved by the Ethics and Research Committee FACENE / FAMENE, the data collection was performed along October of 2015. Data were tabulated and statistically analyzed by using the SPSS statistical software (18th version). The study followed the ethical aspects recommended by the CNS Resolution no. 466/2012, as well as the CFM Resolution 1931/2009, Chapter XII. Results: The survey found that about $50 \%$ students did not have sufficient knowledge on the brain death diagnosis, and $85.5 \%$ is not aware on the National Transplant System; thus implying a negative dynamics in relation to the organ transplantation process. The research results suggest that the institutions should review their curriculum contents on such subjects. Conclusion: The lack of information on this issue makes thousands of professionals are not interested in the area neither get involved in the cause, thus contributing for the loss of viable organs and consequently in the death of people who could be treated.

Keywords: Organ Transplantation; Medicine Student; Nursing Student; Tissue and Organ Harvesting.

\section{REFERÊNCIAS}

1. Freire ILS, Mendonça ILS, Pontes VO, Vasconcelos QLDAQ, Torres GV. Morte encefálica e cuidados na manutenção do potencial doador de órgãos e tecidos para transplante. Revista Eletrônica de Enfermagem. 2012 out/ dez;14(4):903-12.

2. BRASIL. Lei $n^{\circ}$ 9.434, de 4 de fevereiro de 1997.

3. Legislação Federal de Saúde [homepage na internet]. Ministério da Saúde; [acesso em 10 de maio de 2015]. Transplante. Disponível em: http://bvsms.saude.gov.br/bvs/ legislacao/transplantes.php

4. Bitencourt A.G.V, Neves FBCS, Larissa D, Nascimento DT, Neves NMBC, Torreão LA, et al. Avaliação do conhecimento de estudantes de medicina sobre morte encefálica. Revista Brasileira de Terapia Intensiva. 2007 abr/jun;19(2).

5. Conselho Federal de Medicina (CFM) - Código de Ética Médica: Resolução CFM n¹931, de 17 de Setembro de 2009. CFM, 2010.
6. Portal do Médico [homepage na internet]. Brasília: Conselho Federal de Medicina; [acesso em 10 de maio de 2015]. Resolução CFM no 1.480/97. Disponível em: http://www. portalmedico.org.br/resolucoes/CFM/1997/1480_1997.htm

7. Maia. B. O; Amorim. J.S. Morte encefálica: conhecimento de acadêmicos de medicina e enfermagem. JBT J Bras Transpl. 2009;12:1088-91.

8. Galvão FHF, Caires RA, Neto RSA, Mory EK, Figueira ERR, Otsuzi TS, Bacchella T, et al. Conhecimento e opinião de estudantes de medicina sobre doação e transplantes de órgãos. Rev Assoc Med Bras. 2007;53(5) 401-6.

9. Brasil. Conselho Nacional de Educação. Parecer CNE/CES 1133/2001 - Homologado.

10. Neto JAC, Sirimarco MT, Delgado AAA, Lara CM, Lima WG. Estudantes de Medicina da UFJF e doação de órgãos para transplante. HU Revista. 2012 jan/mar;38(1).

11. Reis ML, Popov DCS. Percepção de estudantes de enfermagem sobre a doação de órgãos. Rev Enferm UNISA. 2009;10(2):107-12. 